\title{
HCV/HIV Coinfection: A New Treatment Paradigm
}

\author{
Paul Y. Kwo, Saurabh Agrawal
}

\section{Comment on:}

Sulkowski MS, Naggie S, Lalezari J, et al. Sofosbuvir and ribavirin for hepatitis $\mathrm{C}$ in patients with HIV coinfection. JAMA 2014;312:353-361.

It is estimated that 7 million people are co-infected with human immunodeficiency virus (HIV) and hepatitis C virus (HCV) in the world (J Hepatol 2006;44:S6-9). HIV infection accelerates the progression of $\mathrm{HCV}$, with higher rates of histologic fibrosis and cirrhosis, decompensated liver disease, and death. Until recently, treatment of HCV required the use of interferon-based regimens with long durations of therapy and significant side effects to achieve meaningful sustained virologic response (SVR; N Engl J Med 2006;355:2444-2451). Historically, HCV infection has been more challenging to treat in patients coinfected with HIV owing to the hematologic effects of the therapy, the immune compromised host, and the drug-drug interactions with the first-generation protease inhibitors. It is estimated that $70 \%$ of individuals with HIV/HCV coinfection are not eligible for interferon-based therapies (Clin Infect Dis 2013;56:718-726). Owing to the natural history of HIV/HCV and an accelerated fibrosis rate, the current American Association for the Study of Liver Diseases (AASLD)/Infectious Diseases Society of America (IDSA) guidelines recommend priority treatment for HCV/HIV coinfected patients, regardless of liver fibrosis stage. Historically, SVR rates have been lower in the HIV/HCV coinfected population and they have been thus considered a special population. In 1 study involving 711 patients treated with interferon and ribavirin, an overall SVR rate of $31 \%$ was achieved with the SVR rate being $18 \%$ for genotypes 1 and 4 and 50\% for genotypes 2 and 3. This is markedly lower than non-HIV-infected SVR rates in patients with similar genotypes (Hepatology 2009;50:407-413). In 2011, the first direct-acting antiviral agents (DAAs), NS3/4A serine protease inhibitors, boceprevir and telaprevir were approved for $\mathrm{HCV}$ treatment in conjunction with pegylated interferon and ribavirin. The addition of telaprevir or boceprevir to pegylated interferon and ribavirin improved SVR rates to $74 \%$ and $63 \%$, respectively, in genotype $1 \mathrm{HIV} / \mathrm{HCV}$-infected individuals who were eligible for interferon-based therapy (Ann Intern Med 2013;159:86-96; Lancet Infect Dis 2013;13:597-605). Achieving SVR is important because coinfected patient who achieve SVR have a significant decrease in incidence rates per 100 years of overall mortality, liver-related mortality, and liver decompensation (Hepatology 2009;50:407-413).

In 2013, 2 new DAAs were approved for treatment of HCV. Simeprevir, a protease inhibitor, was approved for patients with genotype 1 in combination with pegylated interferon and ribavirin for 24-48 weeks based on treatment response (Lancet 2014;384:403-413). Sofosbuvir, a nucleotide polymerase inhibitor, was also approved in 2013 in combination with pegylated interferon and ribavirin for 12 weeks for patients with genotypes 1 and 4 and with ribavirin alone for 12-24 weeks in genotypes 2 and 3 . The overall SVR rate with triple combination was 90\% among patients with predominantly genotype 1 and 4 at 12 weeks (Lancet 2014;384:403-413). Genotypes 2 and 3 may achieve SVR rates of approximately $90 \%$ with 12 or 24 weeks of sofosbuvir and ribavirin and do not require interferon ( $\mathrm{N}$ Engl J Med 2013;368:1878-1887; N Engl J Med 2014;370:1993-2001).

This is the author's manuscript of the article published in final edited form as:

Kwo, P. Y., \& Agrawal, S. (2015). HCV/HIV Coinfection: A New Treatment Paradigm. Gastroenterology, 148(7), 1470-1471. http://doi.org/10.1053/i.gastro.2015.04.031 
In the PHOTON-1 trial, the efficacy of an all oral, interferon-free regimen for HCV genotypes 1 , 2, and 3 patients coinfected with HIV was evaluated. This was a multicenter, open-label, nonrandomized, uncontrolled phase III trial involving 34 academic, private practice, and community health centers in the United States and Puerto Rico (JAMA 2014;312:353-361).

A total of 224 patients were included with 115 genotype 1 treatment-naïve patients assigned to receive sofosbuvir and ribavirin for 24 weeks, 68 treatment-naïve patients with genotype 2 or 3 assigned to receive sofosbuvir with weight-based ribavirin for 12 weeks, and 41 patients treatment experienced patients with HCV genotype 2 or 3 assigned to receive sofosbuvir and ribavirin for 24 weeks. The SVR rate in the genotype 1 treatment-naïve group was 76\%. Treatment-naïve patients with HCV genotypes 2 and 3 achieved SVR12 of $88 \%$ and $67 \%$ with 12 weeks of treatment. Patients with treatment experienced genotype 2 and 3 achieved SVR12 rates of $92 \%$ and $94 \%$ after receiving treatment for a total of 24 weeks.

The PHOTON-1 study provides a glimpse at the future of HCV therapy for coinfected individuals as SVR rates were comparable with or superior to interferon-based therapies for genotypes 1,2 , and 3 , with anall oral regimen free of interferon for 12-24 weeks. The inclusion criteria for this study was markedly broader owing to the lack of drug-drug interactions with sofosbuvir compared with other classes, such as protease inhibitors, and included regimens containing emtricitabine-tenofovir in combination with atazanavir ritonavir, darunavir, ritonavir, efavirenz, raltegravir, or orrilpivirin. This lack of interaction of sofosbuvir with the cytochrome P450 3A4 metabolized drugs allowed the administration of this therapy in combination without dose adjustment or adjustment of frequency of dosing which is a major advance of the treatment of HCV in those coinfected with HIV. In the genotype 1 population, the SVR rate of $76 \%$ was comparable with the SVR rate reported with pegylated interferon-based therapies with firstgeneration protease inhibitors and occurred in one-half the duration (24 weeks). Moreover, this SVR rate was achieved in a population that had contraindications to interferon, including psychiatric illnesses, seizure disorders, autoimmune disorders, and other comorbidities that had historically excluded patients from interferon-based therapies for HCV. The SVR rates in genotypes 2 and 3 treatment-naïve and treatment-experienced populations were similar to the SVR rates reported with sofosbuvir and ribavirin when given in non-HIV-infected populations and superior to those reported with interferon-based therapies. Moreover, patients did not require erythropoietin or other support, and the rate of completion in this study was markedly greater than noted in those receiving interferonbased therapies with or without DAAs owing to the excellent safety profile of sofosbuvir, as well as the high barrier to resistance. Indeed, with both telaprevir- and boceprevir-based therapies, therapy was discontinued owing to adverse events as well as development of resistance-associated variants. In the PHOTON-1 study, the resistance-associated variant S282T was not identified in any subject who failed to achieve SVR and those who developed breakthrough had undetectable levels of sofosbuvir, suggesting noncompliance. For genotypes 2 and 3, greater SVR rates were seen when treatment was extended to 24 weeks in the treatment-experienced group, but importantly the therapy was well-tolerated with an additional 12 weeks conferring no increase in adverse events. Patients with genotype 3 treated for 12 weeks showed lesser SVR rates of $67 \%$ as expected given the recommended treatment duration of 24 weeks in those without HIV coinfection.

This is the author's manuscript of the article published in final edited form as:

Kwo, P. Y., \& Agrawal, S. (2015). HCV/HIV Coinfection: A New Treatment Paradigm. Gastroenterology, 148(7), 1470-1471. http://doi.org/10.1053/i.gastro.2015.04.031 
The genotype 1 SVR rates for this population still lag behind what has been reported for non-HIV coinfected HCV patients where SVR rates of $>95 \%$ have been reported using combinations of DAAs with and without ribavirin (N Engl J Med 2014;370:1483-1493; N Engl J Med 2014;370:1889-1898; N Engl J Med 2014;370:1594-1603; N Engl J Med 2014;370:1604-1614; N Engl J Med 2014;370:1973-1982). This study did not include patient with treatment experienced genotype 1, although it is likely that higher rates of SVR will be seen when multiple DAA classes are combined. The PHOTON-1 results have been confirmed in the PHOTON-2 study where SVR rates were $85 \%, 88 \%, 89 \%$, and $84 \%$ among patients with genotype 1, 2, 3, and 4, respectively. This was observed in a population that included genotype 1 nonresponders (Molina et al, Program and Abstracts of the 20th International AIDS Conference, 2014) and safety of this regimen of sofosbuvir and ribavirin for 24 weeks was again confirmed. Even with these high SVR rates, preliminary data using the combinations of DAAs in HIV/HCV-coinfected individuals suggest that SVR rates will be comparable with those achieved without HIV coinfection (Molina et al, Program and Abstracts of the 20th International AIDS Conference, 2014; J Hepatol 2014;60:S7). The CORAL-1 study demonstrates that the combination of paritaprevir, ombitasvir, and dasabuvir with ribavirin may be given to genotype 1-infected HIV patients with an overall SVR rate of $93 \%$ in those receiving atazanavir- or raltegravir-based antiretroviral. A small study has reported high SVR rates with combination of sofosbuvir/ledipasvir in HIV/HCV coinfected individuals receiving tenofovir/emtricitabine plus efavirenz or raltegravir or rilpivirine $(97 \%)$ or no antiretroviral therapy (100\%; Hepatology 2014;60:236A-241A).

\section{Comment}

The PHOTON-1 study heralds the era where special populations, such as HIV/HCV coinfected individuals, will be able to experience the same high SVR rates as those without HIV coinfection. It is likely that sofosbuvir and ribavirin will not be the first line therapy for coinfected individuals, because combinations of DAAs will prove to be more effective. Removing interferon from the therapy is a dramatic leap forward for this population, who can now experience SVR rates higher than ever seen in the interferon era across all genotypes. However, the elimination of ribavirin is also a worthwhile goal, and preliminary data suggest that this too is on the horizon. Finally, although these high SVR rates are encouraging for all clinicians who treat the HIV/HCV-coinfected population, access to therapies worldwide will also be important. This requires a collaborative effort including physicians, governments and policy makers, drug developers, and insurers. Nonetheless, the future of therapy for HIV/HCV coinfected individuals has arrived, and we are on the verge of moving one of the largest special populations of HCV-infected individuals worldwide into the not-so-special category.

This is the author's manuscript of the article published in final edited form as:

Kwo, P. Y., \& Agrawal, S. (2015). HCV/HIV Coinfection: A New Treatment Paradigm. Gastroenterology, 148(7), 1470-1471. http://doi.org/10.1053/i.gastro.2015.04.031 